\title{
Improved Dynamic Modeling and Velocity Loop Augmentation for Quadcopters with estimated Rotor-Drag Coefficient
}

\author{
Youn Tae Kang ${ }^{1}$ and Sung Kyung hong ${ }^{2 *}$ \\ ${ }^{1}$ Research Institute, Young Poong Electronics, 42, Techno 1-ro, Yuseong-gu, \\ Daejeon,34016, Korea \\ ${ }^{2}$ Department of Aerospace Engineering, Sejong University \\ Seoul, 05006, Korea \\ 1'ytkang@ypelec.co.kr, ${ }^{2}$ skhong@sejong.ac.kr
}

\begin{abstract}
This paper proposes a quantitative derivation of the rotor-drag coefficients for the improved quadcopter model, and a velocity control loop augmentation for enhanced maneuverability based on the improved dynamic model. A novel experimental method using accelerometer output is proposed to quantitatively derive key rotor-drag coefficients for the improved dynamic model, In succession, to compensate for the poor (sluggish) maneuverability during attitude control mainly caused by rotor-drag, a velocity controller is augmented as an outer loop to the existing attitude controller. For this control scheme velocity estimators are implemented with accelerometer output as feedback based on the improved dynamic model. The fidelity of the improved dynamic model and the effectiveness of the proposed control scheme were verified in flight tests.
\end{abstract}

Keywords: Quadcopter, Rotor-Drag coefficient, Velocity control, Maneuverability

\section{Introduction}

Recently, quadcopter flying robots (drones) have seen rapid growth with increasing civilian applications including search-and-rescue, tracking, and surveillance [1-2]. This accelerated growth can be attributed to the development of MEMS-based inertial sensors and high-speed BLDC motors, along with the implementation of low-power technology in high- performance micro-controllers. drones, driven by four rotors, are relatively easy to produce and maintain due to their simple structures. With numerous features such as vertical takeoff and landing, hovering, multi-directional movement, and high maneuverability, they can be operated in various areas including indoors, cities with tall buildings, and mountainous regions. However, the small battery capacity and limitations of small BLDC motors impose restrictions on payload and operating time. Despite these current restrictions, drones are emerging as a promising industrial tool thanks to their low production cost, the possibility of further downsizing, and expansion of various civilian applications [1-2].

Drones are difficult to control due to their unstable dynamic characteristics, and several control theories have been developed to date[3-9]. Most studies involve simplified models that neglect rotor drag. These simplified models may be suitable only when the drones are in hovering condition without external interference, but do not account for changes in dynamic characteristics that accompany velocity changes during maneuvering flight. As such, controllers based on simplified models fail to ensure the uniform performance throughout the entire flight conditions, and simulation results tend to be significantly different from an actual flight with drag. To resolve this issue, several studies use improved

Received (December 28, 2017), Review Result (February 26, 2018), Accepted (March 5, 2018)

* Corresponding Author 
dynamic models that include a drag proportional to linear velocity [10-14]. While most research stopped at qualitatively analyzing the influence of drag, more detailed analysis is required such as on the physical basis of drag, and a quantitative derivation of the drag coefficient for the improved dynamic models.

The purposes of this study are:

1 A quantitative derivation of the rotor-drag coefficients for the improved dynamic model

2 A velocity control loop augmentation for enhanced maneuverability based on the improved dynamic model.

For the improved dynamic model, a novel experimental method using accelerometer output is proposed to quantitatively derive key rotor-drag coefficients. In succession, to compensate for the poor (sluggish) maneuverability during attitude control mainly caused by rotor-drag, a velocity controller is augmented as an outer loop to the existing attitude controller. For this control scheme velocity estimators are implemented with accelerometer output as feedback based on the improved dynamic model. The fidelity of the improved dynamic model and the effectiveness of the proposed velocity loop augmented control scheme was verified in flight tests.

\section{Improved Dynamic Model}

\subsection{Without Rotor-Drag Consideration}

In general, the coordinate system used in flight modeling is defined by the inertial frame and body frame. As shown in Figure 1, the inertial frame uses the NED (North-East-Down) coordinate system, while the body frame has the front as the $\mathrm{x}$-axis, the right as the y-axis, and the right-hand rule as the z-axis.

Assuming that the drones frame is rigid-body and that the $x y$ and $y z$ planes are symmetrical (product of inertia $\left(i_{i j}=0, i \neq j\right)$, the simplified 6 DOF equation of motion without rotor-drag considerations is as given in Eq. (1). The velocity component of translational motion $(u, v, w)$ and angular velocity component of rotational motion $(p, q, r)$ fall under the body frame. Using the coordinate transformation matrix $C_{B}^{I}$ and $j_{r}$ shown in Eq. (2) and Eq. (3), they can be converted to the velocity component $(U, V, W)$ and time derivatives of Euler angle $(\dot{\phi}, \dot{\theta}, \dot{\psi})$, respectively.

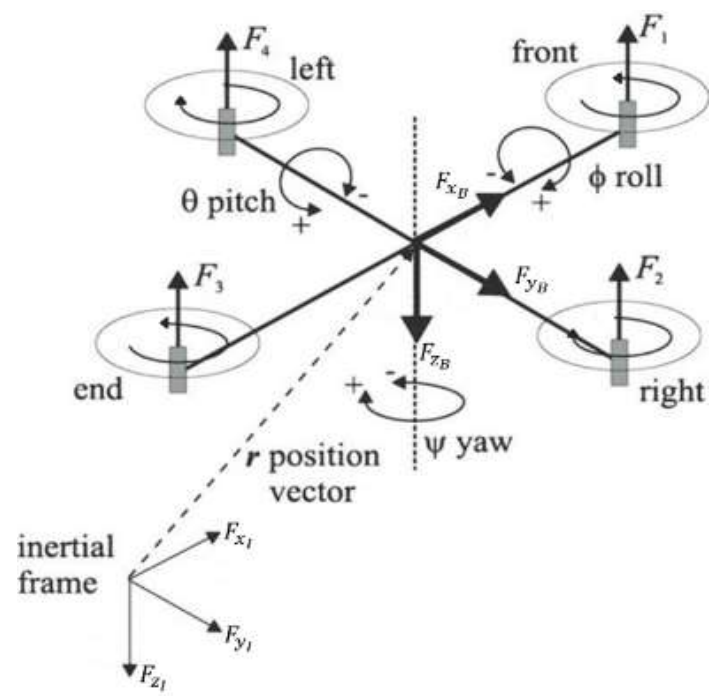

Figure 1. The Coordinate System of Quadcopter 


$$
\begin{aligned}
& \dot{u}=(v r-w q)-g s \theta \\
& \dot{p}=\left(\left(I_{y y}-I_{z z}\right) q r+L\right) / I_{x x} \\
& \dot{v}=(w p-u r)+g s \phi c \theta \\
& \dot{q}=\left(\left(I_{z z}-I_{x x}\right) p r+M\right) / I_{y y} \\
& \dot{w}=(u q-v p)+g c \phi c \theta-F_{t} / m \quad \dot{r}=\left(\left(I_{x x}-I_{y y}\right) p q+N\right) / I_{z z} \\
& C_{B}^{I}=\left[\begin{array}{ccc}
c \theta c \psi & s \phi s \theta c \psi-c \phi s \psi & c \phi s \theta c \psi+s \phi s \psi \\
c \theta s \psi & s \phi s \theta s \psi+c \phi c \psi & c \phi s \theta s \psi-s \phi c \psi \\
-s \theta & s \phi c \theta & c \phi c \theta
\end{array}\right] \\
& J_{r}=\left[\begin{array}{ccc}
1 & s \phi t \theta & c \phi t \theta \\
0 & c \phi & -s \phi \\
0 & \frac{s \phi}{c \theta} & \frac{c \phi}{c \theta}
\end{array}\right]
\end{aligned}
$$

where, $g$ is the gravitational acceleration $\left(9.8 \mathrm{~m} / \mathrm{s}^{2}\right), m$ is the mass of the quadcopter $(\mathrm{kg}), \mathrm{F}_{\mathrm{T}}$ is the total thrust $(\mathrm{N}), \mathrm{I}_{\mathrm{i}}$ is the moment of inertia of each axis $\left(\mathrm{kgm}^{2}\right)$, and $\mathrm{L}, \mathrm{M}, \mathrm{N}$ are moments of each axis $(\mathrm{Nm}) . \mathrm{c}=\cos , \mathrm{s}=\sin$ and $\phi, \theta, \psi$ represent Euler angles (roll, pitch, yaw).

When we consider only longitudinal motion in Eq. (1), the linearized model at the hovering condition is as follows.

$\dot{u}=-g \theta$

$\dot{\theta}=q$

$\dot{q}=\frac{M}{I_{y y}}$

This simplified dynamic model of Eq. (4), which does not consider rotor-drag, exhibits the following inconsistencies during flight.q

When maintaining the constant attitude, velocity (u) does not increase as in Eq. (4), but is maintained in proportion to the angle amplitude.

The output of the accelerometer, used to measure specific force, is not always 0 .

As shown above, the simplified dynamic model(Eq. (4)) is effective only in the hovering condition.

\subsection{With Rotor-Drag Consideration}

This section briefly presents the mathematical basis for the rotor-drag term based on the Blade Element Theory [14-15], and introduces an improved dynamic model incorporates rotor-drag coefficients during translational motion. Here, for the brevity, the high-order term of velocity and acceleration term were considered negligible. The effect of rotor drag on the moment term was also excluded.

When the geometric center of the rotor $\left(A_{i}\right)$ is moving at $\vec{V}_{i}$, and rotor plane are rotating at $\vec{\Omega}$ (perpendicular to the axis of rotation $\vec{k}_{b}$ ), the aerodynamic expression for thrust $\vec{F}_{t}$ arising from rotor rotational speed $\left(\vec{\omega}_{\mathrm{i}}\right)$ is as follows.

$\vec{F}_{i}=-a \vec{\omega}_{i}^{2} \vec{k}_{b}-\vec{\omega}_{i}\left(\lambda_{1} \vec{V}_{A i}-\lambda_{2} \vec{\Omega} \times \vec{k}_{b}\right)$

where, $a$, and $\lambda_{1}$ are positive constants.

When a rigid rotor is assumed, $\lambda_{2}$ can be ignored, and $\vec{\omega}_{\mathrm{i}} \lambda_{1} \overrightarrow{\mathrm{V}}_{\mathrm{A}_{\mathrm{i}}}$ term becomes the 'rotor- drag'. The general model for translational motion, taking into account the thrust of Eq. (5), is as follows.

$$
\begin{aligned}
m \dot{\vec{V}}_{c} & =C_{I}^{B} \vec{F}_{g}+\sum_{i=1}^{4} \vec{F}_{i} \\
& =C_{I}^{B} \vec{F}_{g}-a\left(\vec{\omega}_{1}^{2}+\vec{\omega}_{1}^{2}+\vec{\omega}_{1}^{2}+\vec{\omega}_{1}^{2}\right) \vec{k}_{b}-\lambda_{1}\left(\vec{\omega}_{1}+\vec{\omega}_{2}+\vec{\omega}_{3}+\vec{\omega}_{4}\right)
\end{aligned}
$$


where, $\vec{V}_{c}=\vec{u}_{b}+\overrightarrow{v l}_{b}+w \vec{k}_{b}$ and this is the translational velocity at the center of gravity of the quadcopter, and $C_{I}^{B}$ is the transpose of $C_{B}^{I}$ in Eq. (2).

Just like in Section 2.1, when we consider only longitudinal motion in Eq. (6), the linearized model can be expressed as follows.

$\dot{\mathrm{u}}=-\mu u-\mathrm{g} \theta$

$\dot{\theta}=\mathrm{q}$

$\dot{\mathrm{q}}=\frac{\mathrm{M}}{\mathrm{I}_{\mathrm{yy}}}$

where, $\mu=4 \lambda_{1} \bar{\omega} / \mathrm{m}$, and $\mu\left(\mathrm{s}^{-1}\right)$ represents the rotor- drag coefficient.

\subsection{Estimation of the Rotor-Drag Coefficient}

The output of the accelerometer (measurement of specific force), installed on the drones (body frame), is as follows.

$\overrightarrow{\mathrm{a}}_{\mathrm{m}}^{\mathrm{b}}=\frac{1}{\mathrm{~m}}\left(\overrightarrow{\mathrm{F}}_{\mathrm{T}}-\mathrm{C}_{\mathrm{I}}^{\mathrm{B}} \overrightarrow{\mathrm{F}}_{\mathrm{g}}\right)$

where $\overrightarrow{\mathrm{F}}_{\mathrm{T}}$ is the total external force acting on the drones and $\overrightarrow{\mathrm{F}}_{\mathrm{g}}$ is the gravity.

When a constant thrust $\left(\vec{F}_{t}\right)$ without rotor-drag and angle are maintained as shown in Figure 2, the total external force in the body frame is as follows.

$\vec{F}_{T} \approx-\vec{F}_{t}+C_{I}^{B} \vec{F}_{g}$

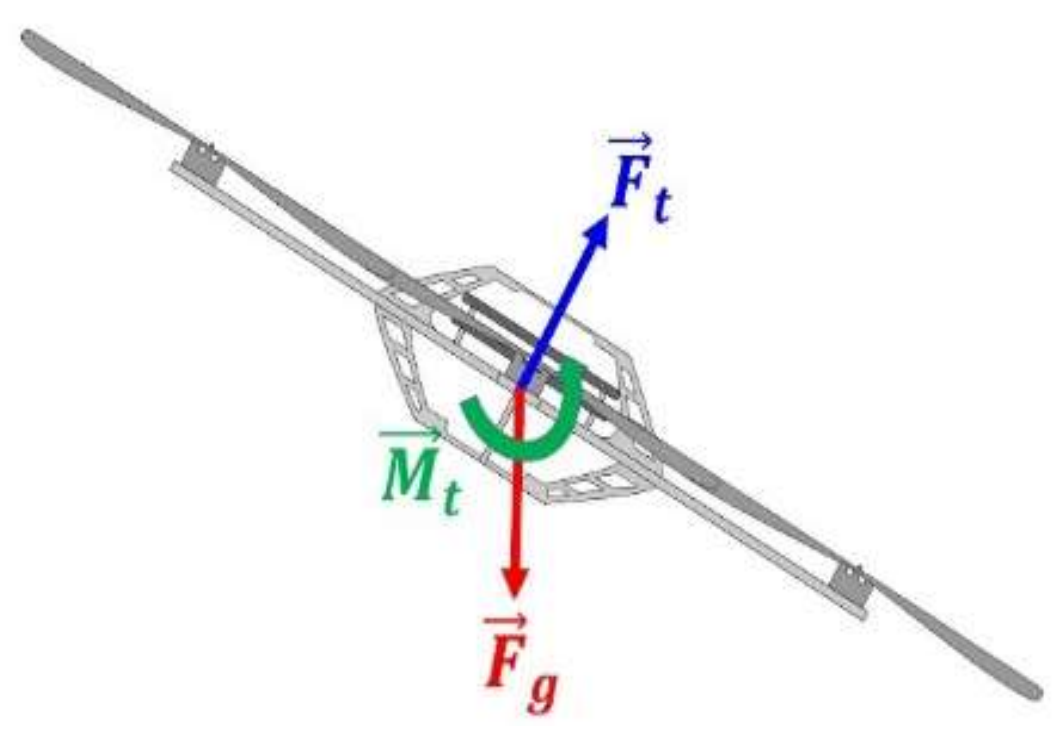

Figure 2. Free-Body Diagram at a Constant Attitude in the Air

Substituting Eq. (9) into Eq. (8), the following equation is obtained for the accelerometer.

$\vec{a}_{i}^{b}=\left[\begin{array}{c}a_{m i} \\ a_{m j} \\ a_{m k}\end{array}\right]=\frac{1}{m}\left[\begin{array}{c}0 \\ 0 \\ -F_{t}\end{array}\right]$

According to Eq. (10), the accelerometer output of the $\mathrm{x}$, the $\mathrm{y}$ axis is always 0 . As mentioned in Section 2.1, this is inconsistent with actual flight.

When the improved dynamic model considering rotor-drag (Eq. (6)) is applied, Eq.10) can be expressed as follows. 
$\vec{a}_{i}^{b}=\left[\begin{array}{l}a_{m i} \\ a_{m j} \\ a_{m k}\end{array}\right]=\frac{1}{m}\left[\begin{array}{l}-\mu u \\ -\mu v \\ -F_{t}\end{array}\right]$

The accelerometer output in the $\mathrm{x}, \mathrm{y}$ axis is equivalent to the corresponding rotordrag. Since the accelerometer output of Eq. (11) is expressed as a velocity proportionate to the drag coefficient, it can be used to estimate the drag coefficient.

\subsection{Experimental Estimation of Rotor Drag Coefficient}

Using the mathematical basis presented in Section 2.3, the drag coefficient of the quadcopter was derived through an actual flight. The experimental setup, estimation results, and verification process are described below.

2.4.1. Quadcopter Platforms: The quadcopter platform used in this study is the Hummingbird model developed by Ascending Technology (AscTec). The Hummingbird's autopilot consists of in two layers: LLP (Low- Level Processor) and HLP (High- Level Processor). The LLP collects and processes various sensor (IMU, GPS, pressure, compass sensor, etc.) information to provide estimates of the orientation and of the velocity vector in an inertial frame, and utilizes the inner loop for attitude control. The HLP collects external sensor information like vision sensors while relying on the outer control loop (see Figure 3) for high- level missions. In particular, HLP provides an efficient Software Development Kit (SDK) that enables users to run their algorithms in Matlab/Simulink. The main specifications of the Hummingbird quadcopter are shown in Table 1 [16].

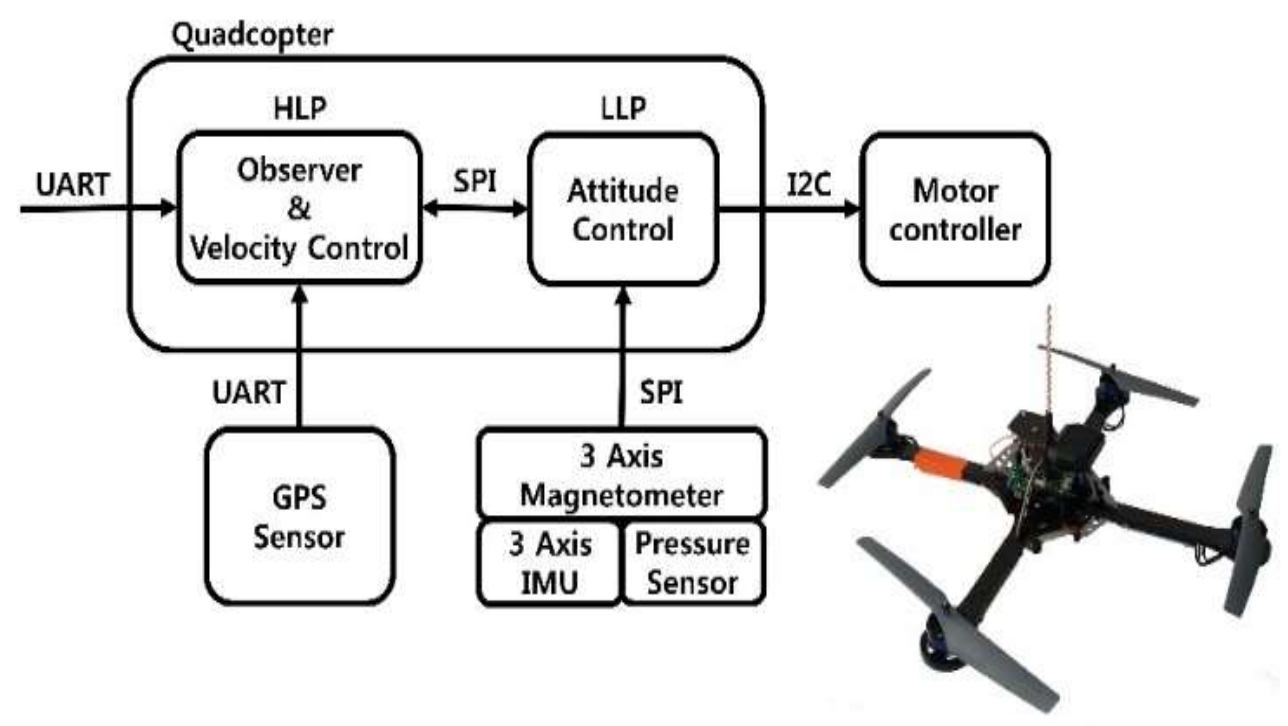

Figure 3. Astec Autopilot

Table 1. Hummingbird Specification

\begin{tabular}{ll}
\hline Specifications & \\
\hline Dimensions & $54 \times 54 \times 5.5 \mathrm{~cm}$ \\
weight & $510 \mathrm{~g}$ \\
Max. payload & $200 \mathrm{~g}$ \\
Max. airspeed & $15 \mathrm{~m} / \mathrm{s}$ \\
Max. flight time & $20 \mathrm{mins}($ non-payload) \\
Battery & $2100 \mathrm{mAh}(\mathrm{LiPo})$ \\
\hline
\end{tabular}


2.4.2. Data Acquisition: Flight experimental data were obtained through the wireless communication module (XBee, Digi ${ }^{\circledR}$ ) between the quadcopter and ground control computer with $50 \mathrm{~Hz}$ update frequency. The collected data include the accelerometer raw measurements $\left(\mathrm{a}_{\mathrm{mi}}, \mathrm{a}_{\mathrm{mj}}\right)$, Euler angles $(\phi, \theta, \psi)$, and velocity vector in an inertial frame $\left(\mathrm{V}_{\mathrm{x}}, \mathrm{V}_{\mathrm{y}}, \mathrm{V}_{\mathrm{z}}\right)$. The velocity vector in body frame $(\mathrm{u}, \mathrm{v}, \mathrm{w})$ is obtained by

$$
\left[\begin{array}{l}
u \\
v \\
w
\end{array}\right]=C_{I}^{B}\left[\begin{array}{l}
V_{x} \\
V_{y} \\
V_{z}
\end{array}\right]
$$

and is considered as the "reference" velocity to validate our modeling assumptions.

The experiment was conducted for 100 seconds over five sessions for estimating the rotor-drag coefficient.

\subsection{Estimation of Rotor Drag $\operatorname{Coefficient}(\mu)$}

The rotor-drag coefficient $\mu$ was extracted by using Eq. (11) based on the accelerometer raw measurements $\left(a_{m i}, a_{m j}\right)$ and the velocity vector in body frame $(u, v, w)$ via trial-anderror. The selected rotor-drag coefficient $(\mu)$ was the average of the estimated ones obtained over five sessions (see Table 2).

Table 2. $\mu$ Estimation Result

\begin{tabular}{cc}
\hline Test No. & $\boldsymbol{\mu}$ Coefficient \\
\hline $\mathbf{1}$ & 0.319 \\
$\mathbf{2}$ & 0.319 \\
$\mathbf{3}$ & 0.322 \\
$\mathbf{4}$ & 0.319 \\
$\mathbf{5}$ & 0.321 \\
\hline \hline Average & 0.320 \\
\hline
\end{tabular}

The simulation results of the improved dynamic model incorporating the estimated rotordrag coefficient were compared with actual flight data. The model used in the simulation is as follows.

$$
\begin{gathered}
\dot{u}_{\theta}=-\mu u_{\theta}-g \theta \\
\dot{v}_{\phi}=-\mu v_{\phi}+g \phi
\end{gathered}
$$

The initial conditions were $\mathrm{u}_{\theta}(0)=\mathrm{u}(0)$ and $\mathrm{v}_{\phi}=\mathrm{v}(0)$.

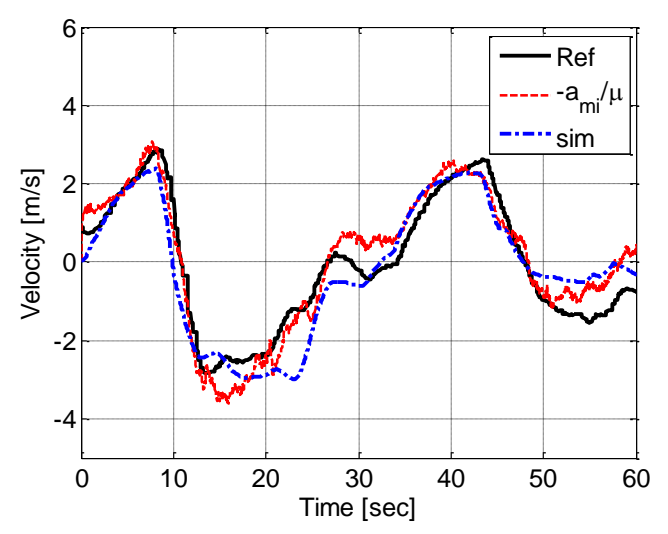

Figure 4. X- axis Velocity Estimation 


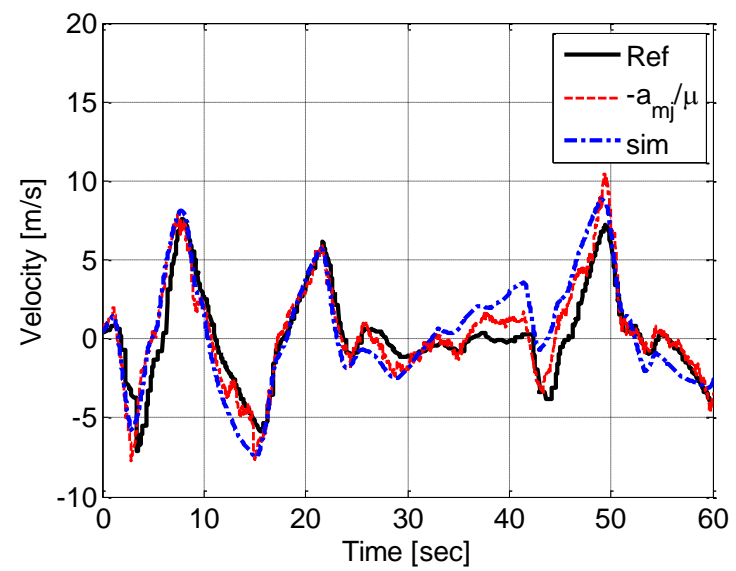

Figure 5. Y- axis Velocity Estimation

Figure 4 shows good agreement between the "reference" velocity u (Eq. (12)), the accelerometer-based velocity $-\mathrm{a}_{\mathrm{mi}} / \mu$ (Eq. (11)), and the simulated velocity $\mathrm{u}_{\theta}$ (Eq. (13)), which reasonably validates our force model. The agreement between the "reference" velocity $\mathrm{v}$, the accelerometer-based velocity $-\mathrm{a}_{\mathrm{mj}} / \mu$, and the simulated velocity $\mathrm{v}_{\phi}$ is also shown in Figure 4.

\section{Velocity Loop Augmentation}

The improved dynamic model, including the rotor-drag coefficient, and the model for the attitude (roll, pitch) controller which is embedded in the LLP were expressed together as shown in Eq. (14). For the model of the built-in attitude controller, a first-order model was assumed with a time constant of 0.11 seconds based on the Prediction Error Method [17].

Figure 5 shows the pitch $(\theta)$ and velocity $(u)$ response to pitch step input for the attitude controller. As expected, the pitch response shows a very fast response $(\tau \approx 0.11 \mathrm{~s})$, while the velocity response shows sluggish response $(\tau \approx 3.2 \mathrm{~s})$ mainly due to rotor-drag.
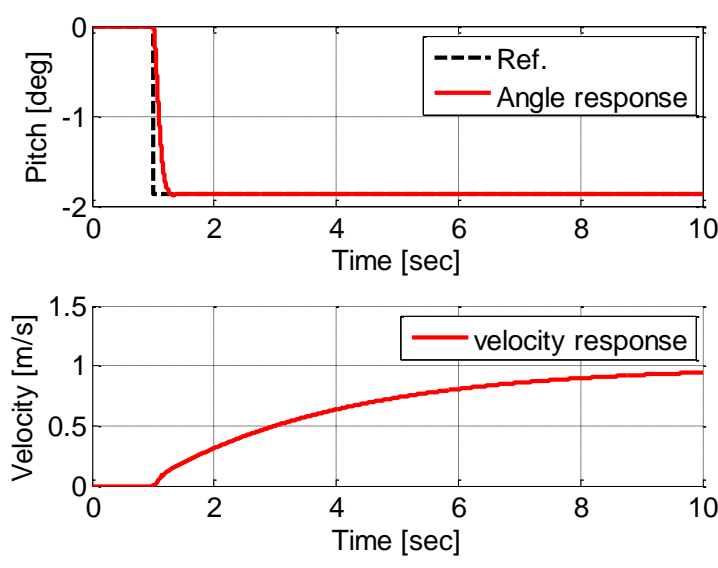

Figure 6. Responses for Attitude Controller (Simulation) 
This leads to poor maneuverability when only attitude controller is involved. On the other hand, when a velocity control loop is augmented as an outer loop to the exiting attitude controller, the maneuverability can be enhanced. For the simplicity, the controller was designed in the form of a PD multi-loop. The control laws are as follows.

$\theta_{c}=K_{2}\left(K_{1}\left(u_{r}-u\right)-\dot{u}\right)$
$\phi_{c}=K_{2}\left(K_{1}\left(v_{r}-v\right)-\dot{v}\right)$
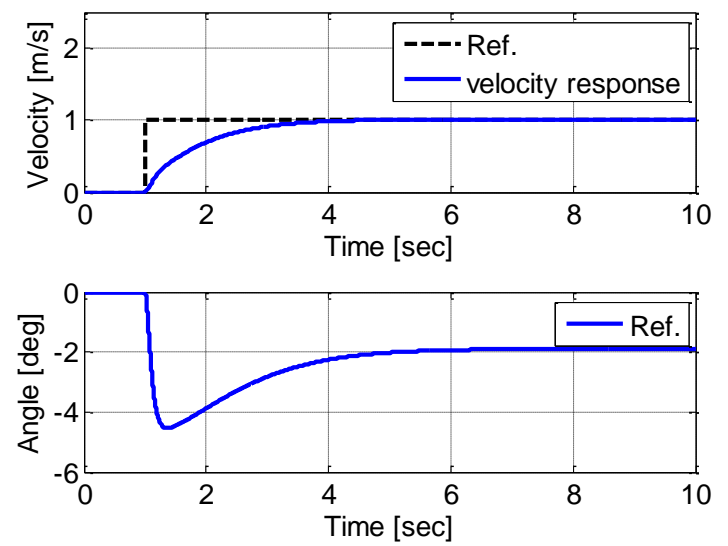

Figure 7. Responses for Velocity Augmented Controller (Simulation)

Figure 7 shows the enhanced maneuverability $(\tau \approx 1$ s) with velocity control loop augmentation.

For this control scheme, velocity state estimators should be implemented. A velocity observer using accelerometer feedback based on the improved dynamic model was designed and implemented in this study. To simplify the system, a fixed gain observer was used. This is not possible under the simplified model without drag considerations.

$\dot{x}=A x+B u, \quad y_{a c c}=C x$

where, $x=\left[\begin{array}{lll}u & v & \theta \phi\end{array}\right]^{T}, u=\left[\theta_{c} \phi_{c}\right]^{T}$

$$
\begin{aligned}
& A=\left[\begin{array}{cccc}
-0.32 & 0 & -9.8 & 0 \\
0 & -0.32 & 0 & 9.8 \\
0 & 0 & -8.74 & 0 \\
0 & 0 & 0 & -9.07
\end{array}\right], B=\left[\begin{array}{cc}
0 & 0 \\
0 & 0 \\
7.54 & 0 \\
0 & 7.28
\end{array}\right] \\
& C=\left[\begin{array}{cccc}
-0.32 & 0 & 0 & 0 \\
0 & -0.32 & 0 & 0
\end{array}\right]
\end{aligned}
$$

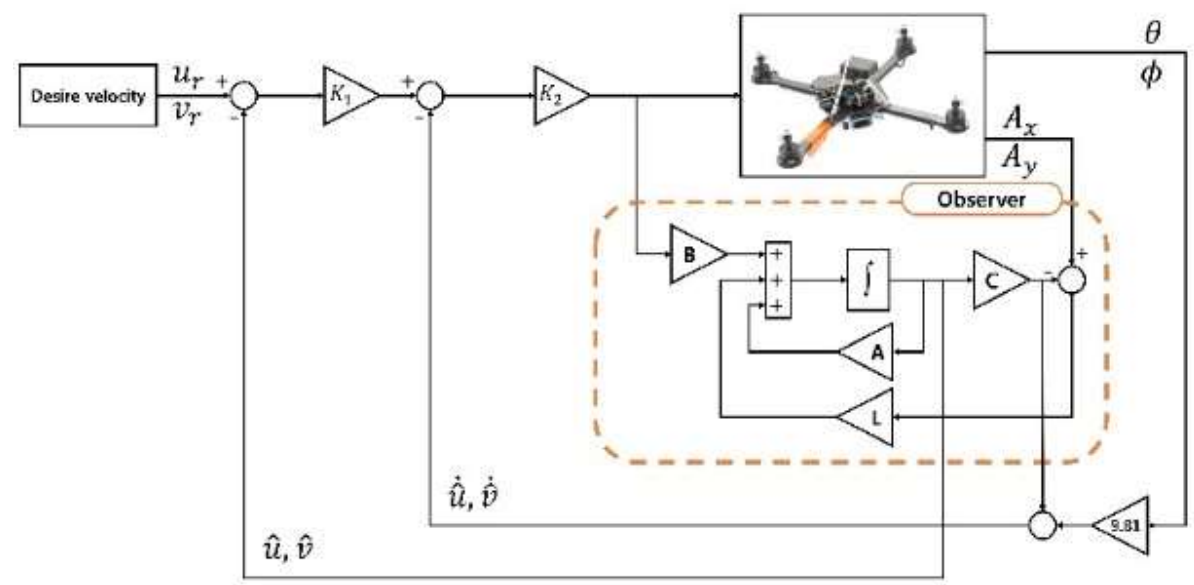

Figure 8. Velocity Control Loop with Observer 


\subsection{Velocity Estimator}

Figure 8 presents the structure of the velocity control loop augmentation with the velocity estimator.

The dynamic equation of the observer is as follows.

$\dot{\hat{x}}=A \hat{x}+B u+L\left(y_{a c c}-C \hat{x}\right)$

where $\hat{x}$ is the estimated state variable and $L$ is the observer gain. We take $y_{a c c}=$ $\left[a_{m i}, a_{m j}\right]^{T}$ as modeled by Eq. (11).

The observer gain $L$ was set to have the fastest response possible using the 'place' function in MATLAB. Because of its simplicity, the linear fixed gain filter is the most practical choice for an embedded processor compared to the other filters involving complex calculations.

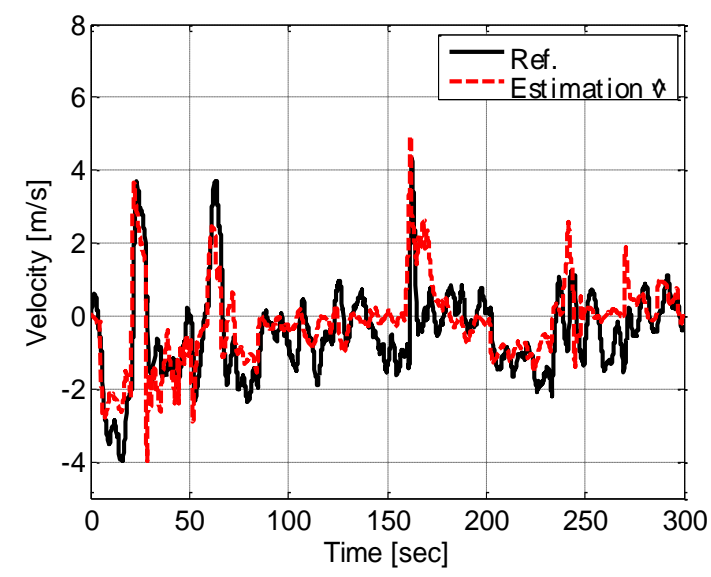

Figure 9. Estimation $\widehat{\boldsymbol{u}}$

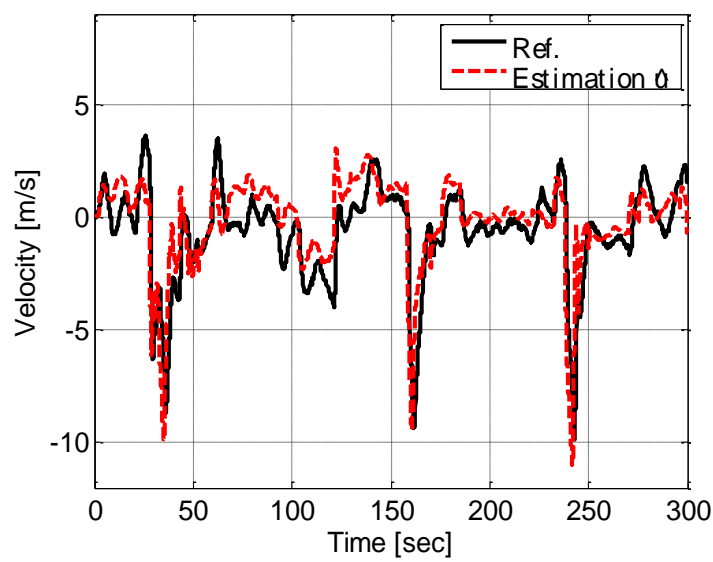

Figure 10. Estimation $\widehat{v}$

Figures $8 \& 9$ show simulation results for the $\mathrm{x}$ and $\mathrm{y}$ body-axis velocity estimation using accelerometer output obtained in actual flight. From the graphs, we can verify that the observer-estimated velocity $(\hat{u}, \hat{v})$ is highly similar to the reference velocity $(u, v)$.

\subsection{Velocity Loop Augmentation}

Using the observer-estimated velocity $(\hat{u}, \hat{v})$, a velocity controller is augmented as an outer loop to the exiting attitude controller to enhance the maneuverability. 
The control laws, modified from Eq. (14), are as follows.

$$
\begin{aligned}
\theta_{c} & =K_{2}\left(K_{1}\left(u_{r}-\hat{u}\right)-\dot{\hat{u}}\right. \\
\phi_{c} & =K_{2}\left(K_{1}\left(v_{r}-\hat{v}\right)-\dot{\hat{v}}\right.
\end{aligned}
$$

where, $\hat{u}, \hat{v}$ and $\dot{\hat{u}}, \dot{\hat{v}}$ represent the estimated velocities and accelerations for each axis.
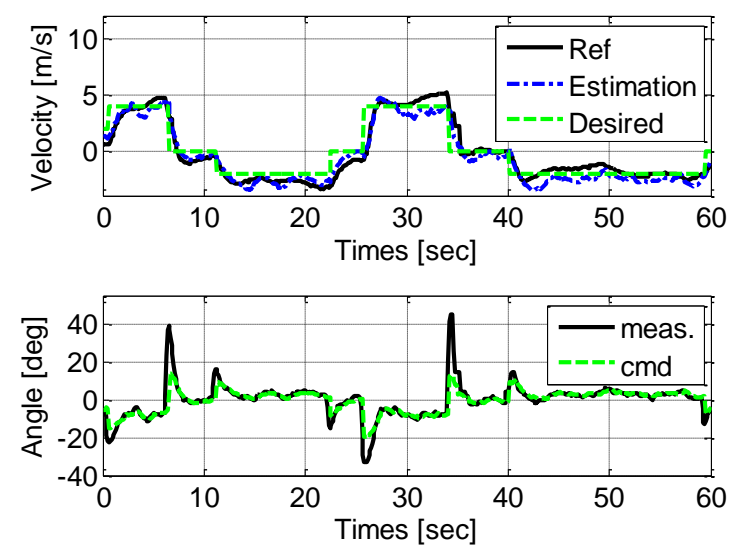

Figure 11. Responses for Velocity Augmented Controller in Flight

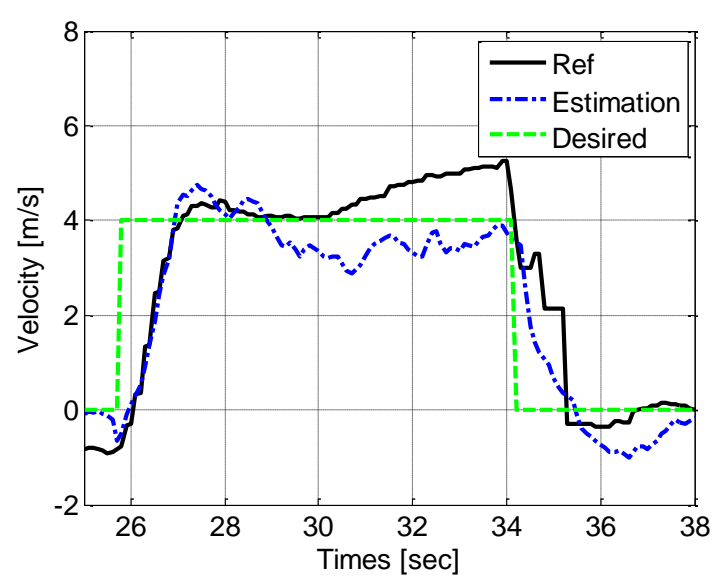

Figure 12. Zoomed Figure 9

As expected from the simulation results in Figure 11, the velocity response shows enhanced maneuverability ( $\tau \approx 1.2 \mathrm{~s}$ ), which is a $50 \%$ improvement compared to that when only the attitude controller is used. Results for the y-axis were similar to the $\mathrm{x}$-axis.

\section{Conclusion}

This paper proposes a quantitative derivation of the rotor-drag coefficients for the improved quadcopter model, and a velocity control loop augmentation for enhanced maneuverability based on the improved dynamic model. Mathematical models and contradictions in actual flight were verified through experiments. And the reference velocity is based on GPS because velocity sensor was absent. The result that controlled the estimated velocity, the maneuverability was improved by about 40-50\%.It'll be an opportunity to increase the efficiency of the position control. Also, the estimated coefficient for a lot of flight and the proposed control scheme were successfully implemented, resulting in a quadcopter not only much faster maneuvering but also easier to fly than with the usual 
scheme. But this paper does not solve the problem of external disturbance because the velocity is estimated based on the acceleration. Future studies will lead to improved results including external environments.

\section{Acknowledgments}

This work was supported by the Korea Institute for Advancement of Technology(KIAT) grant funded by the Korean government(Motie : Ministry of Trade, Industry\&Energy) (No. N0002431).

\section{References}

[1] X. Zhang, X. Li, K. Wang and Y. Lu, "A Survey of Modelling and Identification of Quadrotor Robot", Hindawi Publishing Corporation, Abstract, and Applied Analysis, Volume 2014, Article ID 320526, 16 pages.

[2] J. Moyano Cano, "Quadrotor UAV for wind profile characterization", (2013).

[3] S. Bouabdallah, A. Noth and R. Siegwart, "PID vs LQ Control Techniques Applied to an Indoor Micro Quadrotor", 2004 IEEE/RSJ International Conference on Intelligent Robots and Systems, (2004), pp. 2451-2456.

[4] S. Bouabdallah and R. Siegwart, "Backstepping and Sliding-mode Techniques Applied to an Indoor Micro Quadrotor”, 2005 IEEE International Conference on Robotics and Automation, (2005), pp. 2247-2252.

[5] S. L. Waslander, G. M. Hoffmann, J. S. Jang and C. J. Tomlin, "Multi-Agent Quadrotor Testbed Control Design: Integral Sliding Mode vs. Reinforcement Learning”, IEEE/RSJ International Conference on Intelligent Robots and Systems, (2005), pp. 486-473.

[6] G. M. Hoffmann, H. Huang, S. L. Waslander and C. J. Tomlin, "Quadrotor Helicopter Flight Dynamics and Control Theory and Experiment", AIAA Guidance, Navigation and Control Conference and Exhibit, (2007).

[7] J. P. How, B. Bethke, A. Frank, D. Dale and J. Vian, "Real-Time Indoor Autonomous Vehicle Test Environment”, IEEE Control Systems Magazine, (2008) April, pp. 51-64.

[8] V. Kumar and N. Michael, "Opportunities and Challenges with Autonomous Micro Aerial Vehicles", 15th International Sympossium on Robotics Research, (2011).

[9] M. Hehn and R. D. Andrea, "Quadrocopter Trajectory Generation and Control”, International Federation of Automatic Control World Congress, (2011).

[10] T. Madani and A. Benallegue, "Backstepping Control for a Quadrotor Helicopter", IEEE/RSJ Intl. Conf. on Intelligent Robots and Systems, (2006) October, pp. 3255-3260.

[11] R. Xu and U. Ozguner, "Sliding Mode Control of a Quadrotor Helicopter", IEEE Conf. on Decision and Control, (2006), pp. 4957-4962.

[12] T. Madani and A. Benallegue, "Control of a Quadrotor Mini-Helicopter via Full State Backstepping Technique", IEEE Conf. on Decision and Control, (2006), pp. 1515-1520.

[13] R. C. Leishman, J. Macdonald, R. W. Beard and T. W. McLain, "Quadrotors \& accelerometers state estimation with an improved dynamic model", Control. Syst. Mag., vol. 34, no. 1, (2014).

[14] P. Martin and E. Salaun, "The true role of accelerometer feedback in quadrotor control", 2010 IEEE International Conference on Robotics and Automation.

[15] W. Johnson, Helicopter Theory. Princeton University Press, (1980).

[16] http://wiki.asctec.de/display/AR/AscTec+Hummingbird.

[17] M. Goo Yoo and S. Kyung Hong, "System Identification of the Quadrotor Flying Robot in Hover using Prediction Error Method", Advanced Science and Technology Letters, (Electrical and Eletronic Engineering 2015), vol. 118, pp. 21-26.

\section{Authors}

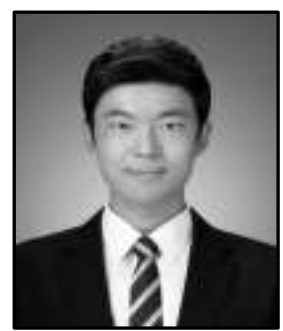

Youn Tae Kang

2013 B.S. at the Department of Mechanical and Aerospace

Engineering of Sejong Univ.

2016 M.S. at the Department of Mechanical and Aerospace

Engineering of Sejong Univ.

2017 Present Senior Research Engineer, Research Institute

Young Poong Electronic CO,. LTD. 


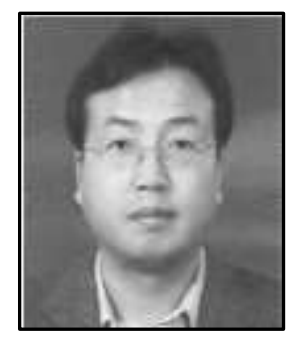

Sung Kyung Hong

1987 B.S. at the Department of Mechanical Engineering of

Yonsei Univ.

1989 M.S. at the Graduate School of Mechanical Engineering of Yonsei Univ.

1998 Ph.D. in Engineering at Texas A\&M Univ.

1989 2000 Senior researcher at the Agency of

Defense Development

$2000 \sim$ present Professor of the Department of

Mechanical and Aerospace

Engineering at Sejong Univ.

Main interest: Design of navigation guidance and control system 